Küster, Bärbel

\title{
Visuelle Kontaktzonen in der Bildenden Kunst, Europa - Afrika
}

Chapter in book | Published version

This version is available at https://doi.org/10.14279/depositonce-7128

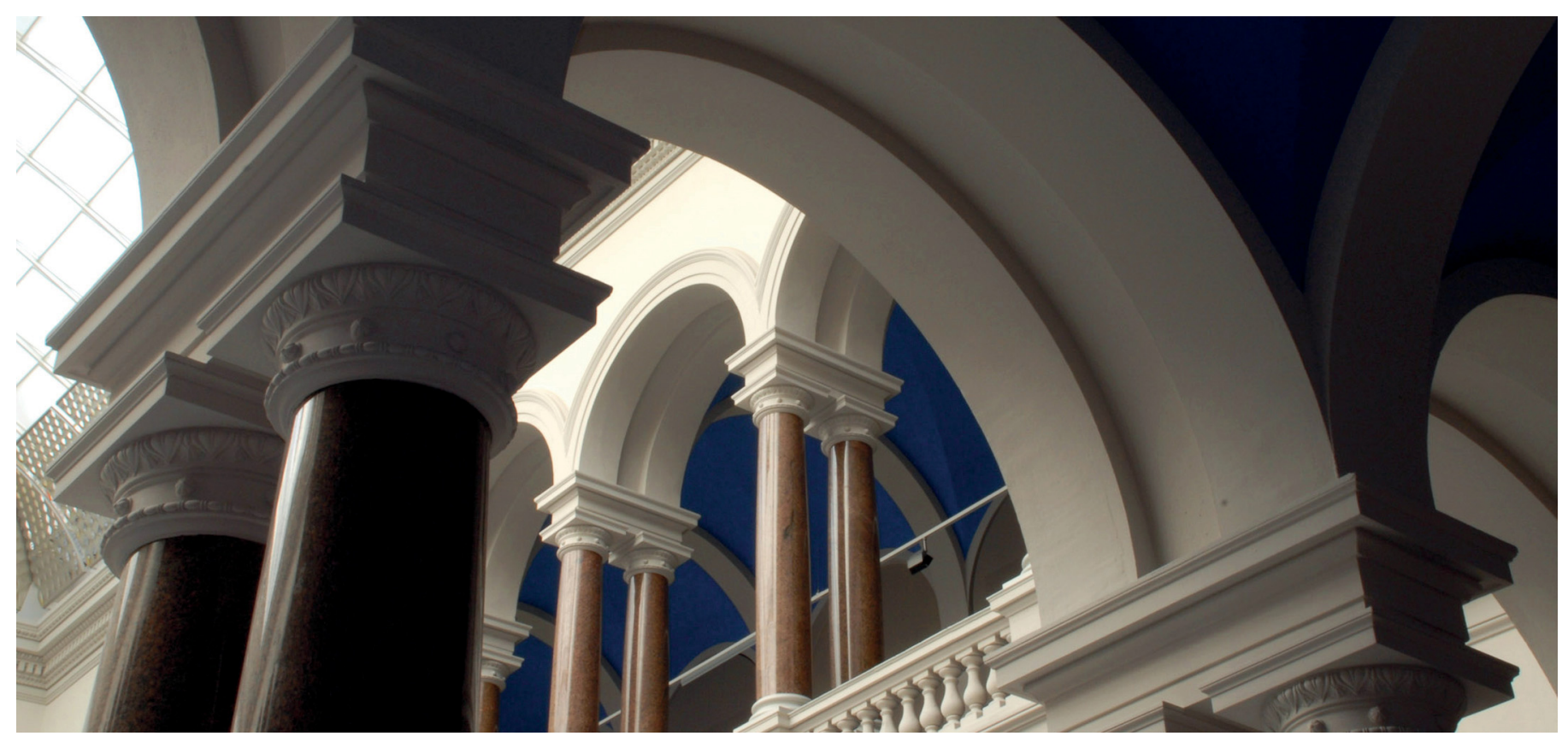

This text was published in Gutberlet, Helff (Eds.), Die Kunst der Migration. Aktuelle Positionen zum europäisch-afrikanischen Diskurs, transcript Bielefeld, 2011. It is posted here by permission of transcript Verlag for personal use only, not for redistribution.

Küster, Bärbel (2011). Visuelle Kontaktzonen in der Bildenden Kunst, Europa - Afrika. In: Die Kunst der Migration. Aktuelle Positionen zum europäisch-afrikanischen Diskurs (pp 201-211). Bielefeld, Transcript. 


\title{
Visuelle Kontaktzonen in der bildenden Kunst, Europa - Afrika
}

\author{
BÄRBEL KÜSTER
}

Visuelle Kontaktzonen zwischen der Kunst der europäischen Moderne und afrikanischen Kunstwerken entstanden über reisende Kunstobjekte, über Reproduktionen, Ausstellungen und durch reisende Künstler. Sie sind in drei oder vier Generationen zu finden. Zugleich korrelieren diese in loser Form auch mit unterschiedlichen Zeitzonen kunsthistorischen Epochenverständnisses; so verstehen afrikanische Kritiker heute zeitgenössische Kunst in Afrika synonym zur Kunst des 20. Jahrhunderts. ${ }^{1}$ $\mathrm{Zu}$ den bedeutendsten Orten, an denen die Begegnung mit europäischer Kunst stattfanden, zählen Kunstschulen in verschiedenen afrikanischen Ländern, an denen zugleich lokale Kunstauffassungen unterrichtet wurden. ${ }^{2}$ Erst in jüngerer Zeit ist die Aufmerksamkeit auf die Geschichte der Kunsterziehung im höheren Ausbildungswesen gelenkt worden. Eine Konferenz des Pan-African Circle of Artists in Nigeria widmete sich 2002

1 Folgt man Nkiru Nzegwu, wird »zeitgenössisch« im Sinne von »modern« verwendet; vgl. das Interview mit Kazeem Adeleke: »Interviewing Professor Nkiru Nzegwu« (o.S. 2005). Das Interview wurde 1999 geführt und erstmals publiziert. Nzegwu ist Professor am Department of African Studies in Binghampton, USA.

2 Es kann hier keine vollständige Auflistung der afrikanischen Kunstschulen gegeben werden. Sie stellen jedoch mit Ausstellungen die zentralen Institutionen für nigerianische Künstler seit Beginn des 20. Jahrhunderts, um sich selbst einen Ort zu geben und auf einem internationalen Kunstmarkt zu verorten. Vgl. Olu Oguibe: Finding a Place. Nigerian Artists in the Contemporary Art World (2002), S. $261 \mathrm{f}$. 
der Rolle des Künstlers in Afrika. ${ }^{3}$ Eines der Ergebnisse der Konferenz war, wie John Picton und Ola Oloidi zusammenfassen, »[ ... ] a clear need for the authoritative documentation of the art media of more recent inception, i.e. since the 1850 s $^{4}$. In dem Maße, wie man sich heute bewusst wird, dass Künstler auch in Afrika eine Rolle bei der Formulierung von Identitäten, des Kulturverständnisses und des politischen Bewusstseins spielen, wurde deutlich, dass das Ausbildungssystem, wie es in den meisten Staaten durch die Kolonialmächte eingeführt wurde, neu konzipiert werden muss. ${ }^{5}$ Vielleicht bedeutet diese beginnende Historisierung auch, dass eine bestimmte Phase der Migration von Künstlern, die sich stark mit westlichen Autoritäten der klassischen Moderne wie Pablo Picasso oder Paul Klee beschäftigten, und der Abbildungen ihrer Werke aus und nach Afrika abgeschlossen ist.

Die Rezeption westlicher Modernismen in Afrika zu würdigen, jenseits einer Interpretation als bloßes Epigonentum, heißt, die hier entstandenen Interpretationen der Moderne gleichermaßen als Tradition ernst zu nehmen wie solche in Europa. Ein Blick auf die Orte der Vermittlung, die Kunstschulen in Afrika, verdeutlicht wie im Brennglas die gegenseitigen Imaginationen zwischen Archaismen und Universalismen. Die Geschichte der bildenden Kunst Afrikas im 20. Jahrhundert über die Kunstvermittlung arbeitet der Utopie einer universellen Kunst zugleich entgegen wie sie ihr auch Argumente zuspielt, weil hier die visuellen Interpretationen kulturell-lokal konkretisiert und differenziert und zugleich gemeinsame Ideen in Vorgängen von Übertragung und Gegenübertragung verständlich gemacht werden können.

Aina Onabolu (1882-1963), der in Nigeria als Vater der modernen Kunst gilt, ${ }^{6}$ machte seine ersten Erfahrungen mit westlicher Kunst als Leser populärer Zeitschriften und religiöser Bücher. Mit zwölf Jahren begann er zu malen und hatte schon einen Status als etablierter (Portrait-)Künstler

3 Reports on the Pan-African Conference on the Status, Role and Working Condition of the Artist in Africa (2002).

4 Reports on the Pan-African Conference, ebd., »Report 1« von John Picton und Ola Oloidi, Nr. 5.

5 Reports on the Pan-African Conference, ebd., »Panel Report 2 « von Margaret Nagawa und Frank Ugiomoh.

6 Vgl. hierzu Everlyn Nicodemus: The Black Atlantic and the Paradigm Shift to Modern Art in Nigeria (2009), der auf verschiedene Titel der nigerianischen Forschung zu Aina Onabolu hinweist, wie zum Beispiel Uche Okeke: »History of Modern Nigerian Art« (1979). 
in seiner Heimatstadt Ijebo, später in Lagos, als er 1918 zur Ausbildung nach Paris fuhr, um dort an der Académie Julian zu studieren, dann am St. John Woods College in London, wo er 1922 sein Diplom erhielt. Onabolu spielt in der Kunstgeschichte Nigerias eine bedeutende Rolle, nicht nur, weil ein 1905 entstandenes Porträt als Geburtsstunde der modernen Kunst in Nigeria dargestellt wird. ${ }^{7}$ Onabolu hat nach seiner Rückkehr aus London die Kunsterziehung seines Landes begründet. Vor dem Hintergrund des Phelps-Stokes-Report, eines großen Erziehungsplanes, wurde erlaubt, ausgebildete Kunsterzieher ins Land zu holen. Der einflussreichste unter ihnen war der Engländer Kenneth C. Murray (19021972). Er förderte ab 1933 in seiner Schule in Ibadan den Unterricht in den Handwerkstechniken ebenso wie auch an den höheren Schulen. Die Ausbildung von nigerianischen Künstlern als Kunstlehrer am Government College, Umuahia, war ein weiterer bedeutender Baustein in der Geschichte der Kunst in Nigeria. Während der Nigerianer Onabolu westliche Kunst unterrichtete, betonte der Engländer Murray die »traditionellen « nigerianischen Techniken ${ }^{8}$ - beendet wurde diese Doppelgleisigkeit durch die Einführung eines für alle weiterführenden Schulen bindenden Curriculums im Rahmen des Cambridge University School Leaving Certificate Examination, ein Abziehbild des englischen Curriculums. Es spielte auch im 1949 eingerichteten Nigerian College of Arts, Science and Technology in Ibadan, Zaria und Enugu eine zentrale Rolle und blieb bis zur Unabhängigkeit 1960 bindend. Mit der Unabhängigkeit rückte auch das nationale Interesse am Kunsterbe Nigerias in den Blick der nigerianischen Regierung, fand in administrativer Hinsicht jedoch erst Niederschlag, als die seit 1966 herrschende Militärregierung 1969 das Nigerian Educational Research Council einberief. In der Nachfolge wurde ab 1973 die Kunsterziehung in einem Dualismus zwischen Traditionalismus und Modernismus eingerichtet. Man kann also davon ausgehen, dass jeder Schüler Nigerias seit 1973 sowohl mit einheimischen wie auch mit westlichen Beispielen der Kunstgeschichte konfrontiert gewesen ist. $^{9}$

7 Olu Oguibe: »Painting and graphic arts«, hier S. 135. Vgl. Grove Art Online unter http://www.oxfordartonline.com [letzter Abruf 25.6.2010].

8 Chidum Onuchukwu: »Art Education in Nigeria« (1994), S. 57.

9 Über die Ausbildungssituation der zweiten bedeutenden nigerianischen Kunstschule, der Nsukka-University informiert der 2002 erschienene Ausstellungskatalog: Simon Ottenberg (Hg.): The Nsukka Artists and Nigerian Contemporary Art, Ausstellungskatalog (2002). 
Ein zweites Beispiel gibt die Margaret Trowell School of Industrial and Fine Arts in Uganda, die unter dem Namen Makerere University School of Art 1937 von Margaret Trowell in Kampala ins Leben gerufen wurde. Trowell (1904-1985) lebte von 1929 bis 1958 in Kenia und Uganda. An der Makerere-Schule hatte sie zum einen die Möglichkeit, jenseits staatlicher Curricula zu arbeiten, da es sich um eine private Schule handelte. Zum anderen lag ihr Interesse von Beginn an in der Förderung ugandischer Techniken und Ästhetiken. Trowell selbst hatte in London an der Slade School Kunst und dann am London University Institute of Education Kunsterziehung studiert. Sie war ausgebildet worden im Umfeld des »child art movement « und Teil einer neuen Generation, die vor allem um die Stimulation des Selbstausdrucks in der Kunst bemüht war; ihr Ziel definierte sie folgendermaßen: »I must put all effort into seeing the visual world through African eyes, and further into trying to understand their spiritual and social attitudes towards their own works of art. ${ }^{10}$ Vor ihrer Abreise aus England jedoch sah Trowell in London noch eine Ausstellung nigerianischer Studenten von Kenneth C. Murray. Und hier schließen sich die Kreise, denn Murray wurde von Aina Onabolu eingeladen in Nigeria zu unterrichten. Das Curriculum Trowells war zwar auf der Basis einer europäischen Kunstschule aufgebaut mit einem vierjährigen Kurssystem aus Malerei, Zeichnung, Bildhauerei und Textilkunst ${ }^{11}$ - anders als in Nigeria wurde aber zum Beispiel darauf verzichtet, Perspektive zu unterrichten, wie Trowell feststellt: »There is certainly no need to foist our conventions, such as perpective, on them. ${ }^{12}$ Andererseits hat Trowell aber neue Medien wie Malerei und graphische Drucktechniken in den Unterricht eingeführt.

In der Zeit, als die Trowell-Schule nach Uganda transferiert wurde (1937-1945), führte sie auch eine »experimental art class« ein, in der zunächst innere Bilder gemalt, und dann gemeinsam Reproduktionen von Kunstwerken aus der griechischen Antike, dem Mittelalter, Asiens und

10 Elsbeth Joyce Court: Margaret Trowell and the Development of Art Education in East Africa (1985), S. 36-37. Das »child art movement« war Teil des in Europa in den 1880er Jahren beginnenden Interesses an Kinderkunst, sogenannter primitiver Kunst und Kunst »fremder« Kulturen allgemein, welche daran die grundlegende menschliche Kreativität und ihre »unverstellten« Produkte jenseits eines akademischen Kunstgeschmacks und zentraleuropäischer Ästhetik schätzten.

11 Elizabeth Harney: In Senghor's Shadow. Art Politics and the Avant-Garde in Senegal (2004), S. 68.

12 Elsbeth Joyce Court: »Margaret Trowell and the Development of Art Education in East Africa« (1985), S. 39. 
auch solche von »African carvings and patterns« betrachtet und diskutiert wurden. Sie betonte in ihrer Autobiographie African Tapestry von 1957, dass die meisten Studierenden die afrikanischen Arbeiten am meisten geschätzt hätten. Auch hier wurde durch die britische Administration in Großbritannien und seinen Kolonien nach 1945 eine Angleichung erzwungen, die die Absolventen einem Londoner Diplom in Fine Arts gleichstellen sollte. Trowell konnte nur mit Hilfe Londoner Kollegen die Inhalte vor einem Zugriff aus London retten. ${ }^{13}$

Im Zusammenhang mit der Négritude-Bewegung steht die Berufung des französischen Amateurkünstlers, Mathematik- und Kunstlehrers Pierre Lods (1921-1988), an die École Nationale des Beaux-Arts nach Dakar, Senegal. Lods hatte zuvor gemeinsam mit Rolf Italiander die Poto-PotoSchule in Brazzaville gegründet und bei einem Kongress in Rom einen Vortrag über seine Unterrichtsmethoden gehalten, wo Léopold Sédar Senghor ihn hörte und nach Dakar einlud. Auch Lods verfolgte das unbedingte Ziel einer »afrikanischen Authentizität« und einen weitgehend offenen Unterrichtsstil. ${ }^{14}$ Neben ihm waren Iba Ndiaye (1928-2008) und Papa Ibra Tall (geboren 1935) die wichtigsten Lehrer der École de Dakar. Iba Ndiaye war von der europäischen Kultur geprägt, hatte im Senegal und dann in Montpellier und Paris Architektur und Kunst studiert und war durch Europa gereist, bevor er 1959 wieder nach Dakar kam. Für ihn war - anders als für Pierre Lods - die afrikanische Kunst weltoffen und nicht in einer vermeintlichen Authentizität gefangen, was 1966 zum Bruch mit Lods führte und Ndiaye zur Rückkehr nach Paris bewegte.

Valentin Yves Mudimbe weist auf eine weitere Kunstschule hin, die Pierre Romain-Desfossés im damaligen Elizabethville, heute Lubumbashi, Demokratische Republik Kongo, in den 1950er Jahren gründete. ${ }^{15}$ In seinem Atelier »Le Hangar« (Académie d'Art Populaire) wollte RomainDesfossés die Malerei aus Katanga fördern, indem er ein ästhetisch Unbewusstes seiner Schüler anzusprechen versuchte, welches er in einem »asiatic complex« mit einem »nilotic etiquette« sah. Diese Begriffe, die einem dumpfen vorurteilsgeladenen afrikanischen bzw. asiatischen »Primitivismus« zuarbeiten, sieht Mudimbe für Romain-Desfossés wie auch

13 Vgl. ebd., S. 38-39.

14 Vgl. Harney, In Senghor's Shadow, a.a.O., S. $65 f f$.

15 Vgl. auch Joseph-Aurélien Cornet (Hg.): 60 ans de peinture au Zaïre (1989). 
Frank McEwens Arbeit in Salisbury in Rhodesien (heute Harare, Simbabwe) getragen von einem Ursprünglichkeitsideal, das Letzterer wiederum als »mexican period « oder auch präkolumbianisch bezeichnete. ${ }^{16}$ Mudimbe führt diese reichlich unklaren Vorstellungen zurück auf C. G. Jungs Archetypenlehre. ${ }^{17}$ Die Archetypenlehre kann man als eines von vielen möglichen Konzepten verstehen, Authentizität zu produzieren und die verschiedenen Kultur-Zitate auf eine gemeinsame visuelle Basis (in diesem Fall phylogenetischer) Prägung zu stellen. Die genannten Kulturen werden von Europa aus als »primitive« verstanden, jenseits ihrer konkreten Realität. Sie verschaffen in dieser Vorstellung visuell Zugang zum (Mythos des) Archaischen, an dem in den Augen der genannten Kunstlehrer in besonderem Maße auch Künstler in Afrika partizipierten. Der Anspruch der modernen Künstler in Europa auf Universalität konkurrierte gewissermaßen mit dieser quasi als biologisch imaginierten Nähe zu einem universellen Archaischen. Das Primitivismuskonzept wiederum, also die Idee einer besonderen Nähe afrikanischer Künstler zu einer (nicht näher definierten) Ursprünglichkeit, und damit die Nähe zu einer zutiefst menschlichen Kreativität, für die unter anderem die afrikanische Kunst stehen sollte, wurde mit den Kunstlehrern nach Afrika getragen.

\section{Euromodernismus}

Der nigerianische Historiker, Philosoph und Kunstkritiker Nkiru Nzegwu schlug vor, die in Paris und andernorts entstandene Kunst der klassischen Moderne als Derivate der afrikanischen Kunst zu verstehen und somit die Hierarchisierungstendenzen des Primitivismus einfach umzukehren. Dementsprechend ist der Modernismus der Picassos und Klees von ihm als »Euromodernismus«, eine Spielart der Moderne, bezeichnet worden. ${ }^{18}$ Zwar kann man die Bedeutung der afrikanischen Kunst sicherlich nicht derart ins Zentrum rücken, ${ }^{19}$ aber die Relativierung der Moderne

16 Valentin Y. Mudimbe: »Reprendre, Enunciations and Strategies in Contemporary African Arts« (1999), S. 32f.

17 Vgl. ebd., S. 33.

18 Kazeem Adeleke: »Interviewing Professor Nkiru Nzegwu« (2005).

19 Unter den Kunstwerken, die zum Beispiel die Künstler des Blauen Reiter im Almanach von 1912 belehnten, waren ebenso japanische Holzschnitte, Bronzen aus Benin, bayerische Hinterglasmalerei wie malayische Holzfiguren und gotische Grabmäler. Vgl. Wassily Kandinsky/Franz Marc (Hg.): 
hat ihren Sinn genau darin, die Absolutheitsansprüche dieser Kunstströmung, die weite Teile des 20. Jahrhunderts dominierte, abzulegen. Okwui Enwezor spricht von »großen und kleinen Modernen«, um die Heterotopien und vor allem auch die Ungleichzeitigkeiten zu umschreiben. Die kleinen Modernen sind die Exporte (er spricht hier von »quotations«) aus der europäischen Moderne, die sich an anderen Orten, innerhalb anderer Geschichten weiterentwickelten. ${ }^{20}$ Und: Enwezor kappt den Faden einer universal gültigen Moderne, $\gg[\ldots]$ there is no red line running from modernism to contemporary art«, wertet die lokalen Entwicklungen auf und fordert, den Modernismus radikal auf kleinste Einheiten herunterzubrechen, "we need then to provincialise modernism « ${ }^{21}$

Inzwischen hat sich eine pluralistische Sicht der Moderne durchgesetzt - auch wenn zum Beispiel Rosalind Krauss, Hal Foster, Benjamin H. Buchloh und Yve-Alain Bois in ihrem College Art Reader Art since 1900 den Modernismus als strikt euro-amerikanische Erscheinung verstehen. ${ }^{22}$ In Publikationen wie Kobena Mercers Cosmopolitan Modernisms sind die kulturspezifischen Interpretationen lateinamerikanischer, indischer, afrikanischer und afroamerikanischer Künstler genauer untersucht worden. ${ }^{23}$ Eine weitere Studie, die diesen Prozess anhand eines afrikanischen Künstlers nachzeichnet, ist Sylvester Okwonudu Ogbechies Monographie über den nigerianischen Künstler Ben Enwonwu (1921-1994) und die Inkorporation der europäischen Moderne in eine Philosophie der Igbo, in ihre künstlerischen Traditionen und Wissenssysteme. ${ }^{24}$

Toma Muteba Luntumbue, Kunstkritiker, Künstler und Lehrer für Kunstgeschichte in Brüssel, fasste jüngst die Situation für heutige Künstler aus Afrika so zusammen: »Ihr Werk gehört der zeitgenössischen Kunst

Der Blaue Reiter (Neuausgabe von Klaus Lankheit, 1984/2000). Auch in der französischen Primitivismus-Debatte herrschte eine solche Bandbreite rezipierter Kulturen, vgl. Bärbel Küster: Matisse und Picasso als Kulturreisende. Primitivismus und Anthropologie um 1900 (2003).

20 Okwui Enwezor: »Modernity and Postcolonial Ambivalence« (2009), S. 25. Enwezor folgt hier im wesentlichen Dipesh Chakrabarty: Provincializing Europe, Post-colonial Thought and Historical Difference (2007) und ders.: Habitations of Modernity; Essays in the Wake of Subaltern Studies (2002).

21 Enwezor: »Modernity and Postcolonial Ambivalence«, ebd., S. 28.

22 Rosalind Krauss u.a.: Art since 1900. Modernism, Antimodernism, Postmodernism (2004).

23 Kobena Mercer (Hg.): Cosmopolitan Modernisms (2005).

24 Sylvester Okwunodu Ogbechie: Ben Enwonwu. The Making of an African Modernist (2008). 
an, aber diese Zugehörigkeit ist eingeschrieben in den Anspruch einer multiplen und flexiblen Identität, angesiedelt in einer transnationalen Gleichzeitigkeit, in der alle kulturellen Bezüge breit gefächert sind. «²5

Die Kunst der Moderne ist sowohl in der Ausbildung an europäischen Akademien als auch in der Praxis heutiger Künstler stark in den Hintergrund getreten, ihre Rolle relativiert und ihr universaler Anspruch längst gebrochen - an deutschen Kunsthochschulen spielt beispielsweise der Kubismus wenn überhaupt nur noch eine marginale Rolle. Dennoch gehört er zum kulturellen Erbe und dem kollektiven Gedächtnis. Gerade für Künstler, die sich mit Fragen der Identität beschäftigen, könnten diese historischen Kontaktzonen und die verschiedenen Generationen nicht nur europäischer Kunstrezeption von ebenso großem Interesse sein, wie die Phasen der Auseinandersetzung mit afrikanischer Kunst und Kunstkonzeptionen in Europa für hiesige Künstler.

\section{Medialität, Materialität und Übermittlung}

Für Künstler heute stellt sich das Problem der Appropriation in einer anderen Form. Die Aneignungsprozesse sind weniger von direkten kolonialen Hierarchien geprägt als vielmehr von unterschiedlichen Zugängen zur globalisierten Welt vor dem Hintergrund einer alltäglich-realen, partikularen Erfahrung. Sie bleibt in der Ausbildungssituation an konkrete Vermittler wie an Medien gebunden. Die Medien der Übermittlung haben sich von den schlechten Autotypien der Wochenpresse vom Anfang des 20. Jahrhunderts bis zum Internetauftritt in ihrer materiellen Qualität stark gewandelt. Aber die Frage, ob und welches Bildmaterial für Anregung und Austausch künstlerischer Ideen benutzt wird, bleibt eine ganz konkrete.

Als Beispiel mag hier noch einmal ein Blick zurück dienen: Die künstlerische Farbgebung vieler Kunstwerke der 1950er Jahre ähnelt sich in der matten Farbpalette und der starken Abtönung zu Schwarz hin. Man kann darin ein Echo der Farbgebung schlechter Drucke aus der damaligen Reproduktionstechnik wiedererkennen - sei es bei Pablo Picasso, Georges Rouault, Christian Rohlfs einerseits oder bei Ben Enwonwu, Ahmed Cherkaoui, Erhabor Ogieva Emokpae und Gazbia Sirry anderer-

25 Toma Muteba Luntumbue: »Verschränkte Aneignungen. Künstlerische Dialoge zwischen Afrika und dem kolonialen Europa« (2006), S. 60. 
seits. ${ }^{26}$ Sie wie auch die europäischen Künstler hatten ihre Bildbände und Postkartensammlungen, anhand derer sie sich fremde, alte und zeitgenössische Kunst ansahen. ${ }^{27}$ Ebenso wie in Europa, rezipierten Künstler in Afrika Fotos und Reproduktionen von Kunstwerken. Diese mediatisierte Prägung zeithistorischer Stile, jenseits der Frage, ob Globalisierung oder Lokalisierung, jenseits der eigentlich selbstverständlichen Rezeption anderer Kunstwerke in Europa wie in Afrika, stellt eine eigene Dimension in der Forschung über Kontaktzonen dar. Welche eigene Ästhetik die technische Übersetzung des Bildes hervorgebracht hat, bleibt eine Frage, die noch zu erforschen ist. Ebenso könnte man in der heutigen Perspektive danach fragen, welche Rolle die zeitgenössischen Medien in der kulturellen Begegnung und in der Ausbildung von Studierenden tatsächlich spielen, und welche ästhetischen Konsequenzen die Auseinandersetzung mit digitalen Bildmedien auch interkulturell hat. Das konkrete Trägermaterial künstlerischer Auseinandersetzung über Archive oder vielleicht auch Interviews zu rekonstruieren, könnte ein weiterer Schlüssel sein für die Aufarbeitung einer Geschichte der gegenseitigen Rezeption und Interpretation, deren Bezugspunkte auf die Moderne und die nachfolgenden Kunstkonzepte nicht mit einem »post-« ad acta zu legen sind.

\section{Literatur}

Adeleke, Kazeem: »Interviewing Professor Nkiru Nzegwu«, in: Africa Resource, http://www.africaresource.com, geposted am 12. 5. 2005 [letzter Abruf 10.6.2010].

Adenaike, Tayo: »Between Two Careers«, in: Simon Ottenberg (Hg.): The Nsukka Artists and Nigerian Contemporary Art, Ausstellungskatalog, Washington: National Museum of African Art 2002, S. 123-131.

Chakrabarty, Dipesh: Provincializing Europe: Post-colonial Thought and Historical Difference, Princeton: Princeton University Press 2007.

Chakrabarty, Dipesh: Habitations of Modernity; Essays in the Wake of Subaltern Studies, Chicago: University of Chicago Press 2002.

26 Vgl. z.B. die Abbildungen in Okwui Enwezor (Hg.): The Short Century. Independence and Liberation Movements in Africa 1945-1994, Ausstellungskatalog (2001).

27 Vgl. Küster, Matisse und Picasso, a.a.O. und Dorothy Kosinsky (Hg.): The Artist and the Camera (2000). 
Cornet, Joseph-Aurélien (Hg.): 60 ans de peinture au Zaïre, Ausstellungskatalog, Brüssel: Éditeurs d'Art Associés 1989.

Court, Elsbeth Joyce: »Margaret Trowell and the Development of Art Education in East Africa«, in: Art Education, 38, 6, Nov. 1985, S. 35-41.

Enwezor, Okwui (Hg.): The Short Century. Independence and Liberation Movements in Africa 1945-1994, Ausstellungskatalog, Berlin: Martin-GropiusBau 2001.

Enwezor, Okwui: »Modernity and Postcolonial Ambivalence«, in: Nicolas Bourriaud (Hg.): Altermodern, Ausstellungskatalog, London: Tate Britain, Tate Triennial 2009, S. 27-40.

Harney, Elizabeth: In Senghor's Shadow. Art Politics, and the Avant-Garde in Senegal, Durham: Duke University Press 2004.

Kandinsky, Wassily/Franz Marc (Hg.): Der Blaue Reiter (1912), Neuausgabe v. Klaus Lankheit, München/Zürich: Piper (1984) 2000.

Kosinski, Dorothy (Hg.): The Artist and the Camera. Degas to Picasso, Ausstellungskatalog, Dallas: Museum of Art 2000.

Krauss, Rosalind/Hal Foster/Yve-Alain Bois/Benjamin H. Buchloh: Art since 1900. Modernism, Antimodernism, Postmodernism, London/New York: Thames \& Hudson 2004.

Küster, Bärbel: Matisse und Picasso als Kulturreisende. Primitivismus und Anthropologie um 1900, Berlin: Akademie Verlag 2003.

Luntumbue, Toma Muteba: »Verschränkte Aneignungen. Künstlerische Dialoge zwischen Afrika und dem kolonialen Europa«, in: Marjorie Jongbloed (Hg.): Entangled. Annäherungen an zeitgenössische Künstler aus Afrika, Köln: Volkswagen Stiftung 2006, S. 22-60.

Mercer, Kobena (Hg.): Cosmopolitan Modernisms, Cambridge/Mass./London: MIT Press 2005.

Mudimbe, Valentin Yves: »Reprendre, Enunciations and Strategies in Contemporary African Arts«, in: Olu Oguibe (Hg.): Reading the Contemporary. African Art From Theory to the Marketplace, London: Institute of International Visual Arts 1999, S. 30-47.

Nicodemus, Everlyn: »The Black Atlantic and the Paradigm Shift to Modern Art in Nigeria«, in: Critical Interventions, 3/4, 2009, S. 7-20.

Ogbechie, Sylvester Okwunodu: Ben Enwonwu. The Making of an African Modernist, Rochester: University of Rochester Press 2008.

Oguibe, Olu: »Painting and Graphic Arts«, in: Dictionary of Art, hg. v. Jane Turner, Artikel »Nigeria«, S. 128-139, New York: Grove 1996, S. 135-137. Oguibe, Olu: »Finding a Place. Nigerian Artists in the Contemporary Art World«, in: Simon Ottenberg (Hg.): The Nsukka Artists and Nigerian Con- 
temporary Art, Ausstellungskatalog, Washington: National Museum of African Art 2002, S. 257-278.

Okeke, Uche: »History of Modern Nigerian Art«, in: Nigeria Magazine, Lagos, 128/129, 1979, S. 100-118.

Onuchukwu, Chidum: »Art Education in Nigeria«, in: Art Education, 47, 1, Jan. 1994 (»Art International«), S. 54-60.

Ottenberg, Simon (Hg.): The Nsukka Artists and Nigerian Contemporary Art, Ausstellungskatalog, Washington: National Museum of African Art 2002.

»Reports on the Pan-African Conference on the Status, Role and Working Condition of the Artist in Africa«, in: IJELE, 4, 2002.

Trowell, Margaret: African Tapestry, London: Faber 1957.

Udechukwu, Obiora: „Of Nightsoilmen, Refugees, and Politicians: Or, Peculiar Situations, Peculiar Responses«, in: Simon Ottenberg (Hg.): The Nsukka Artists and Nigerian Contemporary Art, Ausstellungskatalog, Washington: National Museum of African Art 2002, S. 100-116. 